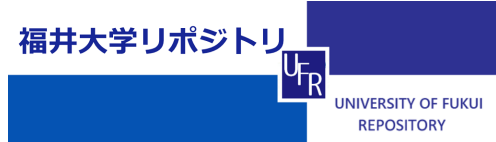

El ect r ochemi cal I y i nst ant aneous reduct i on of conduct i ngpol yani I i ne- coat ed I at ex parti cl es

\begin{tabular}{|l|l|}
\hline $\begin{array}{l}\text { j our nal or } \\
\text { publ i cat i on titl e }\end{array}$ & $214 \mathrm{t}$ Neet ing of the El ect rochemical Soci ety \\
\hline page range & 219 \\
\hline year & 2008 10 \\
\hline URL & ht t p: //hdl . handl e. net /10098/5213 \\
\hline
\end{tabular}




\section{Electrochemically instantaneous reduction of conducting polyaniline-coated latex particles}

Han Chen, Jingyuan Chen, Koichi Aoki

Department of Applied Physics, University of

Fukui, 3-9-1 Bunkyo, Fukui-shi, 910-8507 Japan

\section{Introduction}

Redox latex particles are macromolecules with functionality arising from redox moieties. They can make up uniform polymeric films with large areas by being spread on electrodes. A strategy for making latex redox sites react completely is to use conducting polymers in the electrical conducting state, which can keep the whole particle of equal potential. When the conducting polymeric particle comes into contact with the electrode, it acquires the same potential as the electrode. Then the electrode reaction occurs over the entire particle, and hence the reduction for the conducting-to-insulating conversion is so rapid as to be controlled by the supply of dopant until the path of any conducting site to the electrode is cut off by electric percolation. However, the electrochemical oxidation for the insulating-to-conducting conversion is controlled by the propagation of the conducting zone at the velocity of ca $1 \mathrm{~mm} \mathrm{~s}^{-1}$ in dopant-rich solutions, and is slower than the reduction. This slow oxidation is expected to exhibit the size-dependence of partial electron transfer. This presentation reports the voltammetric conversion of polyaniline-coated polystyrene latex particles dispersed in aqueous acid solution in order to find not only a relationship between the partial reaction and particle size, but also the irreversibility of the conversion.

\section{Experimental}

Styrene was distilled under low pressure, and stored in a refrigerator before use. Seed particles were prepared by the dispersion polymerization of styrene under various conditions depending on particle size. Aniline hydrochloride was added to the suspension of polystyrene latex. The mixture was stirred in an ice bath for $2 \mathrm{~h}$ in order to adsorb aniline onto the surface of the latex. Polymerization was performed by adding $2.45 \mathrm{~g}$ ammonium persulfate (oxidant) to the mixture, which was stirred for 5 $\mathrm{h}$ at $0{ }^{\circ} \mathrm{C}$ and for a further $14 \mathrm{~h}$ at room temperature. The color of the suspension changed from white to dark-green. The suspension was sedimented in a refrigerated centrifuge, SRX-201 (TOMY, Tokyo), to prevent degradation of polyaniline

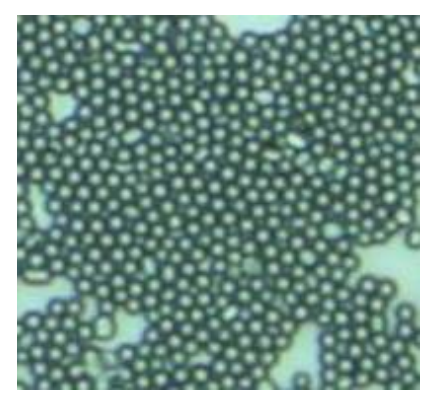

(Fig.1)

Fig.1 Photograph of the suspension by the SEM.

\section{Results and Discussion}

PANI-PS latex particles with uniform size-distribution were synthesized by adjusting amounts of surfactants and varying the stirring rate of the reaction mixture. Microscopic observation of THF-dissolved particles revealed that the latex had a core-shell type. The thickness of PANI-shell, which was evaluated by weight analysis and DTA, was approximately proportional to the radius of the particle. Figure 2 shows logarithmic dependence of $m$ ( PANI-units per particle) and $t$ (thickness of the PANI shell) on the radii. Both variations approximately fell on each line. The slopes of $\log \mathrm{m}$ and $\log \mathrm{t}$ vs. $\log \mathrm{r}_{\mathrm{L}}$ were 3.1 and 1.1, respectively. The proportionality of the amount of PANI per particle with the particle volume suggests a uniform distribution of PANI over the sphere rather than the core-shell structure. Although this fact seems to suggest a uniform PANI-distribution rather than a core-shell structure, it is due to the synthetic conditions for the amount of aniline loaded and the diameter of the core. A quarter of the loaded aniline formed the PANI shell. The thickness of PANI shell was ca. $5 \%$ of the radius.

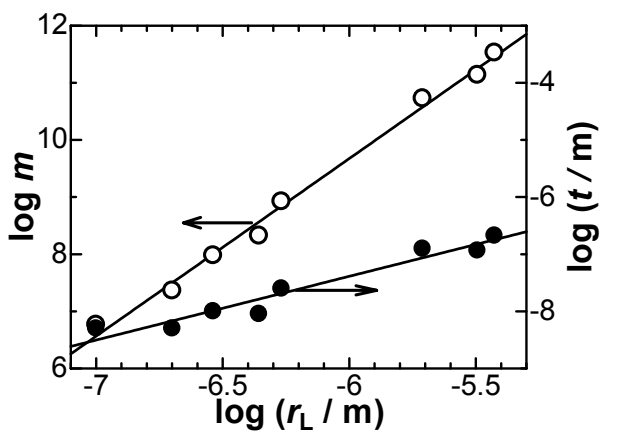

Fig.2. Logarithmic variations of the number of polyaniline-units, $m$, and the thickness of the polyaniline-shell, $t$, with the diameters of various PANI-PS latexes.

The PANI-PS suspension showed a cathodic wave and a very small anodic wave. The cathodic current, which we ascribed to the reduction of emeraldine to leucoemeraldine, was controlled by diffusion of the suspended latex particles rather than adsorbed particles. Values of $I_{\mathrm{p}} v^{-1 / 2}$ were proportional to $r_{\mathrm{L}}^{-0.48}$. This trend was explained in terms of the proportionality of $I_{\mathrm{p}}$ to $D^{1 / 2}$ or $\left(k_{\mathrm{B}} T / 6 \pi \eta r_{\mathrm{L}}\right)^{1 / 2}$ through the Stokes-Einstein equation. The peak currents for various radii agree with the variation in Eq. (2).

$$
\left(-I_{\mathrm{p}} v^{-1 / 2}\right)\left(m c^{*}\right)^{-1}=0.446 F A \sqrt{D F / R T}
$$

That is, $n$ was independent of the radii, different from the voltammograms of a ferrocene-coated suspension. The reduction efficiency in Fig 3, as high as 80-90\%, is ascribed to the electric conducting state of PANI, the inner potential of which can equalize over the shell to the electrode potential when the particle comes into contact with the electrode. In contrast, the oxidation efficiency, as low as non-detection, is ascribed to the slow propagation of the conducting zone.

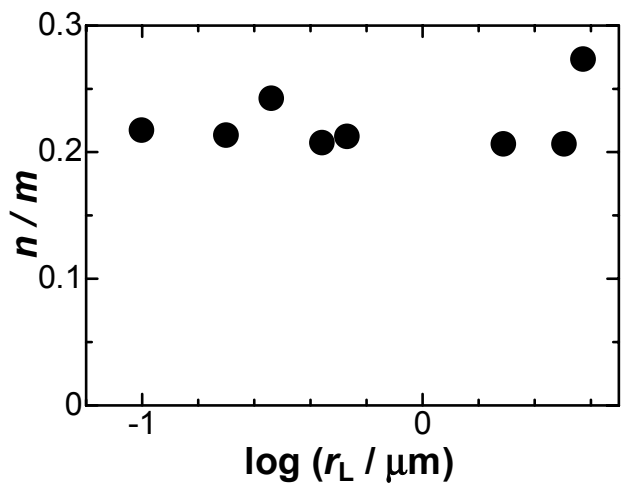

Fig. 3. Variation of $n$ (the number of exchanged charge of PANI per particle) $/ m$ (the number of aniline units per particle) with the radii of the particles. 